\title{
Interferometric Polarimetry of the Cosmic Microwave Background: Methodology
}

\author{
S.T. Myers ${ }^{a}$, J.L. Sievers ${ }^{b}$, J.R. Bond ${ }^{b}$, C.R. Contaldi ${ }^{c}$, \\ B.S. Mason ${ }^{\mathrm{d}}$, T.J. Pearson ${ }^{\mathrm{e}}$, and A.C.S. Readhead ${ }^{\mathrm{e}}$, \\ for the CBI collaboration. ${ }^{\mathrm{f}}$ \\ ${ }^{a}$ National Radio Astronomy Observatory, P.O. Box O, Socorro, NM 87801 \\ (Corresponding author e-mail: smyers@nrao.edu) \\ ${ }^{\mathrm{b}}$ Canadian Institute for Theoretical Astrophysics, 60 St. George St., Toronto, ON \\ M5S 3H8, Canada \\ ${ }^{\mathrm{c}}$ Theoretical Physics, Blackett Laboratory, Imperial College, London SW'7 2BZ, \\ $U K$ \\ d National Radio Astronomy Observatory, 520 Edgemont Road, Charlottesville, VA \\ 22903-2475 \\ ${ }^{\mathrm{e}}$ Astronomy, Caltech 105-24, Pasadena, CA 91125 \\ ${ }^{\mathrm{f}}$ http://astro.caltech.edu/ tjp/CBI/
}

\begin{abstract}
Interferometry has long been used in radio astronomy to enable imaging of astronomical sources with angular resolutions exceeding the diffraction limit of a single aperture. In the past decade, interferometry of the CMB has been carried out with instruments such as the CBI, DASI and VSA which exploited the inherent instrumental stability and simplicity of ell-space analysis of interferometer data. The practice of interferometric polarimetry has been particularly well-developed in the radio astronomical community and DASI and CBI were able to measure the polarization of the CMB over the multipole range $200<\ell<1500$. In this talk, I discuss the theory of interferometry and the mathematics of CMB interferometric polarimetry, using the recent polarization observations of the Cosmic Background Imager (CBI) as an example. Topics will also include description of the data pipeline, handling of contaminating signals and sources, and the construction of optimal maps. I will conclude with the possibility of future CMB interferometers with kilo-element arrays and mega-pixel imaging.
\end{abstract}

Key words: cosmology, observation, cosmic microwave background, polarization 


\section{Introduction}

Interferometry has a venerable history in radio astronomy as a technique to synthesize apertures (1) larger than those constructible as single telescopes, providing higher resolution at the cost of surface brightness sensitivity. Early searches for Cosmic Microwave Background (CMB) anisotropies using generalpurpose radio interferometers started in the late 1970's using the Green Bank Interferometer (2) and then moved to the Very Large Array (VLA) (3, 4). Later pioneering efforts included the use of ATCA (5) and IRAM (6). However, by the late 1980's it was clear that compact arrays with smaller apertures observing at higher frequencies are needed to reach the sensitivity levels required to detect the weak CMB fluctuation signals. For example, (7) used a SIS mixer based interferometer at $43 \mathrm{GHz}$ to place further upper limits. In the 1990's, breakthroughs in the development of low-noise microwave amplifiers provided the technology needed to improve sensitivity, and starting with the Cambridge Anisotropy Telescope (CAT) (8), a number of interferometers have since been constructed to observe CMB anisotropies. In particular, the Cosmic Background Imager (CBI) (9), the Degree Angular Scale Interferometer (DASI) (10), and the Very Small Array (VSA) (11) have provided high quality measurements of CMB anisotropies and the CMB angular power spectrum, and in the case of DASI and CBI, the polarization power spectrum. The BIMA array equipped with a $30 \mathrm{GHz}$ HEMT system(12) was also used to measure the CMB power spectrum at high $\ell$. Other special-purpose interferometers like MINT (13) have pushed towards higher frequencies.

In this contribution, we describe the methodology used to describe the process of interferometric imaging and determination of the angular power spectrum of the CMB. We then describe the pipeline developed for the CBI analysis. We close with some speculation on the future of CMB interferometry. Science results from the CBI are presented in a companion talk by Tim Pearson.

\section{Interferometric Imaging and Polarimetry}

In the following description of the interferometric imaging process, we have adapted the discussion and notation used in (14) and Myers et al. (in preparation). We have tried to keep the notation the same, but have made some changes in order to accommodate polarization and not confuse the reader with multiple uses of the symbols $P, Q$, and $V$ in particular! There are a number of books that can serve as references on the theory and techniques of radio interferometry, for example (1).

We now consider the specific case of an interferometer such as the CBI or DASI 
where the elements (antennas or horns) are aligned in a plane pointed along the direction normal to this plane, or array axis. The signals from pairs of elements are brought together, preserving phase, and multiplied in the correlator. This is schematically shown in Figure 1. For our planar array, the delays along each path to the correlator are compensated so that an initial wavefront impinging on the array from the array axis arrives in phase at each multiplier. Since ideal antenna optics are also designed to bring this wavefront to the focus in-phase, our interferometer effectively computes the cross-correlation of the wavefronts from the sky distribution of brightness over the planes of the apertures of the pairs of elements.

A point source (at infinite distance) in the pointing direction of the array produces wavefronts that are perpendicular to the normal to the array, thus all the correlations have constant amplitude and zero net phase. We calibrate the system such that this constant amplitude is equal to the flux density in Janskys (Jy) ${ }^{1}$ of such a point source. As illustrated in Figure 1, wavefronts coming from direction at angle $\theta$ to the array normal will have a phase gradient over the aperture plane of the array, with the relative phase of a correlation growing linearly with the baseline, or distance between the two antennas in the correlated pair. If we denote the angular offset of a source direction from the reference direction of the array as a vector $\boldsymbol{\theta}$ (in a Cartesian coordinate system in a plane projected onto the sky at the array normal direction), then this phase is given by $2 \pi \boldsymbol{\theta} \cdot \boldsymbol{B}_{i j} / \lambda$ where $\boldsymbol{B}_{i j}$ is the baseline vector between the pair of antennas $i$ and $j$ and $\lambda$ is the observing wavelength. Note that the choice of direction for $\boldsymbol{B}_{\boldsymbol{i j}}$ (ie. from antenna $i$ to antenna $j$ or visa versa) is a convention of the interferometer system that must be carried through the data reduction. Furthermore, this phase factor looks like the phase of a Fourier transform, and indeed the interferometry equations can be formulated in terms of Fourier transforms of the sky brightness projected onto tangent planes (1).

The imaging equations: We can set up the interferometric imaging problem as a linear operation relating the time-ordered correlations or "visibilities" $\tilde{\boldsymbol{v}}$ to the signal $\tilde{\boldsymbol{s}}$

$$
\tilde{\boldsymbol{v}}=\tilde{\mathbf{A}} \tilde{\boldsymbol{s}}+\tilde{\boldsymbol{n}}
$$

where $\tilde{\boldsymbol{n}}$ is the instrumental noise. We use the tilde on vectors and operators to denote them as being in the Fourier plane. The Fourier domain signal $\tilde{\boldsymbol{s}}$ is related to the sky brightness signal $\boldsymbol{s}$ by a Fourier transform $\boldsymbol{s}=\mathbf{F} \tilde{\boldsymbol{s}}$. The utility of this will become apparent when we show the interferometry equations below. We also treat the data and signal vectors as real, although they are most naturally formulated as complex quantities. In this case, the

$\overline{11 \mathrm{Jy}}=10^{-26} \mathrm{~W} \mathrm{~m}^{-2} \mathrm{~Hz}^{-1}$. 


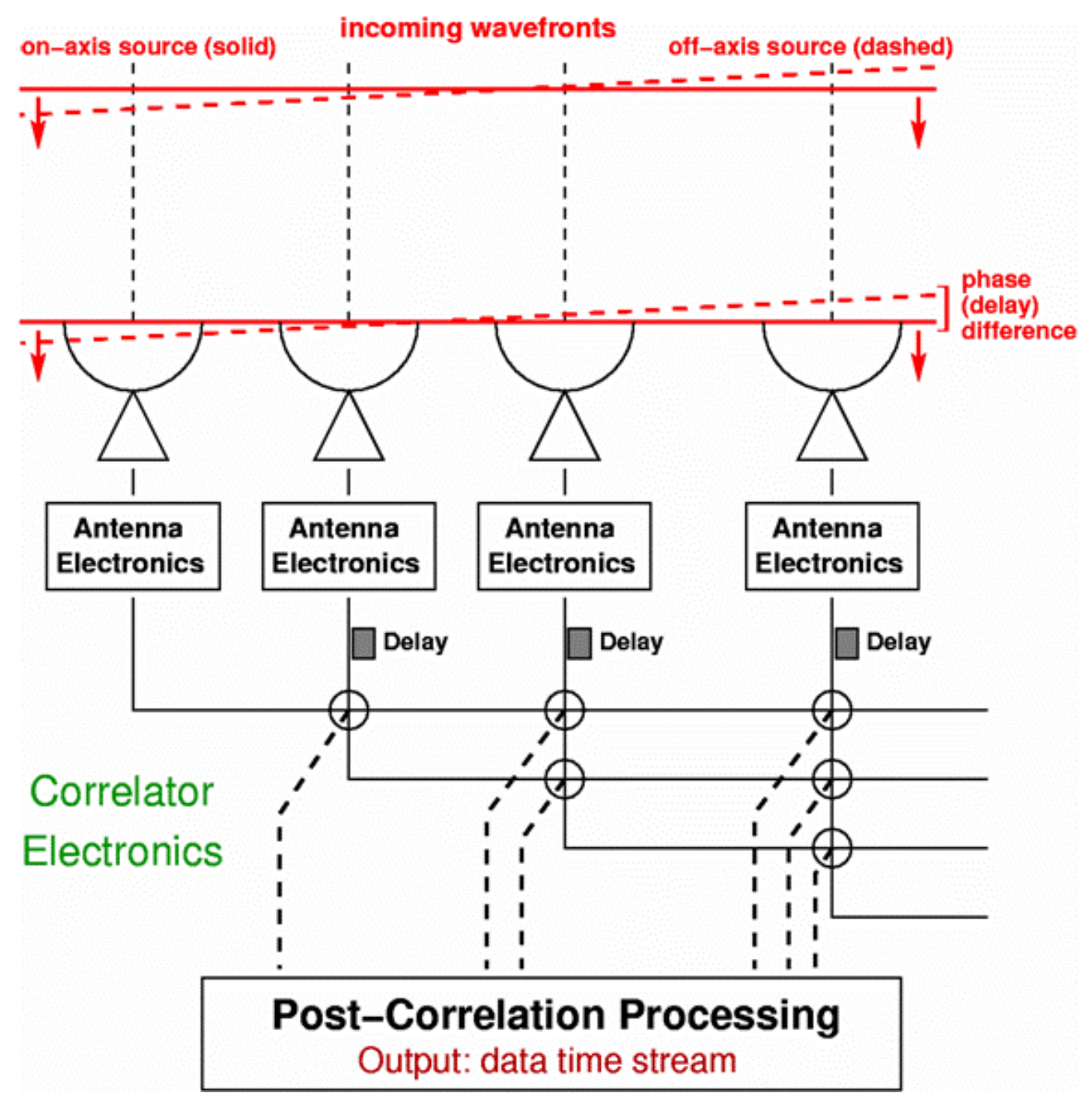

Fig. 1. Schematic of a planar interferometer such as the CBI. Signals from pairs of elements are correlated, with the delays set such that the signals from on-axis wavefronts arrive coherently at the multipliers. Wavefronts coming from an angle $\theta$ off-axis correlate with a residual phase $2 \pi \theta B / \lambda$ between antenna pairs with projected baseline $B$ at observing wavelength $\lambda$. The real and imaginary parts of the complex correlations are computed, and the outputs are complex un-calibrated "visibilities".

real and imaginary parts can be packed into the real vector with the transform symmetries built into the kernels for the operators. We will switch between real quantities in the matrix notation and complex quantities where convenient, so beware.

We further assume that $\tilde{\boldsymbol{n}}$ is a realization of Gaussian noise with zero mean and covariance $\tilde{\mathbf{N}}=\left\langle\tilde{\boldsymbol{n}} \tilde{\boldsymbol{n}}^{\mathrm{t}}\right\rangle$. The probability distribution function for the noise 
is thus

$$
\mathcal{L}=\left(\operatorname{det} \tilde{\mathbf{N}}^{-1} / 2 \pi\right)^{1 / 2} \exp \left[-\frac{1}{2}(\tilde{\boldsymbol{v}}-\tilde{\mathbf{A}} \tilde{\boldsymbol{s}})^{\mathrm{t}} \tilde{\mathbf{N}}^{-1}(\tilde{\boldsymbol{v}}-\tilde{\mathbf{A}} \tilde{\boldsymbol{s}})\right]
$$

which is known as the "likelihood" function in Bayesian statistics. By maximizing $\mathcal{L}$, one can construct the maximum likelihood estimate (MLE) $\tilde{\boldsymbol{m}}_{M L E}$ for the signal $\tilde{\boldsymbol{s}}$, e.g. (15),

$$
\tilde{\boldsymbol{m}}_{M L E}=\tilde{\mathbf{R}}_{M L E}^{-1} \tilde{\mathbf{A}}^{\mathrm{t}} \tilde{\mathbf{N}}^{-1} \tilde{\boldsymbol{v}} \quad \tilde{\mathbf{R}}_{M L E}=\tilde{\mathbf{A}}^{\mathrm{t}} \tilde{\mathbf{N}}^{-1} \tilde{\mathbf{A}}
$$

In effect, we convolve the data using $\tilde{\mathbf{A}}^{\mathrm{t}}$, then deconvolve using $\tilde{\mathbf{R}}^{-1}$, with $\tilde{\mathbf{N}}^{-1}$ as the familiar inverse noise variance weighting factor. In general, the operator $\tilde{\mathbf{R}}$ is ill-conditioned (and singular) as it involves convolution by $\tilde{\mathbf{A}}$ and has incomplete support due to the limited Fourier-space sampling of baselines by the interferometer (see below). In radio interferometry, deconvolution is an arcane art as one tries to find approximate and/or iterative methods to arrive at an appropriate map $\tilde{\boldsymbol{m}}$ that can be transformed into an image $\boldsymbol{m}=\mathbf{F} \tilde{\boldsymbol{m}}$.

We can consider $\tilde{\boldsymbol{m}}_{M L E}$ as a special case of a general "map"

$$
\tilde{\boldsymbol{m}}=\tilde{\mathbf{R}}^{-1} \tilde{\boldsymbol{d}} \quad \tilde{\boldsymbol{d}}=\tilde{\mathbf{H}} \tilde{\boldsymbol{v}}=\tilde{\mathbf{R}} \tilde{\boldsymbol{s}}+\tilde{\boldsymbol{n}}_{\boldsymbol{d}} \quad \tilde{\mathbf{R}}=\tilde{\mathbf{H}} \tilde{\mathbf{A}} \quad \tilde{\boldsymbol{n}}_{\boldsymbol{d}}=\tilde{\mathbf{H}} \tilde{\boldsymbol{n}}
$$

where $\tilde{\mathbf{H}}_{M L E}=\tilde{\mathbf{A}}^{\mathrm{t}} \tilde{\mathbf{N}}^{-1}$. Note that no more information is contained in $\tilde{\boldsymbol{m}}$ (or $\boldsymbol{m}$ ) than in $\tilde{\boldsymbol{d}}$. Therefore, one need only convolve the data with a kernel that approximates $\tilde{\mathbf{H}}_{M L E}$ to be close to the optimal solution. The $\tilde{\boldsymbol{d}}$ can then be used for further processing and analysis. The information on the CMB is contained in the signal covariance $\mathbf{S}=\left\langle\tilde{\boldsymbol{s}} \tilde{\boldsymbol{s}}^{\mathrm{t}}\right\rangle$. This propagates through to the covariance of $\tilde{\boldsymbol{d}}, \mathbf{C}^{S}=\tilde{\mathbf{R}} \mathbf{S} \tilde{\mathbf{R}}^{\mathrm{t}}$.

One can make a "dirty" (ie. not deconvolved) image $\boldsymbol{d}$ of the sky by transforming

$$
\boldsymbol{d}=\mathbf{F} \tilde{\boldsymbol{d}}=\mathbf{R} s+\boldsymbol{n}_{\boldsymbol{d}} \quad \mathbf{R}=\mathbf{F} \tilde{\mathbf{R}} \mathbf{F}^{-1} .
$$

The matrix $\mathbf{R}$ encodes the mapping from the true sky $\boldsymbol{s}$ to the image $\boldsymbol{d}$. For example, the vector formed from the diagonal of $\mathbf{R}$ corresponds to the "primary beam" of the array, relating a unit input sky pixels $s_{i}$ to an output pixel $m_{i}$, while the point spread functions (PSF) are a set of vectors taken from the rows of $\mathbf{R}$ normalized by the diagonal.

We now determine these operators for our interferometer observing the CMB.

Interferometric Polarimetry: Since radio receivers are sensitive to the orthogonal polarizations of the electromagnetic waves, our correlator will pro- 
duce products $p q$ of these, where usually $p q=R R, L L, R L, L R$ for circularly polarized receiver systems (such as the VLA or CBI), or $p q=X X, Y Y, X Y, Y X$ for a linearly polarized system. These are then mapped to the Stokes parameters $I, Q, U, V$ that describe the radiation field. See the series of papers (16), (17), (18) for a detailed description of radio interferometric polarimetry.

For the CMB temperature anisotropy field $T$, we use the correlations sensitive to Stokes $I$, in our case $R R$ and $L L$. For no circular polarization $V=0$ (ie. the CMB), these are equal and we can consider these to be "temperature" visibilities $\tilde{\boldsymbol{v}}_{T}$. Since the wavefronts are summed and correlated, we find in a noiseless system the complex "visibility" (indexed by $k$ )

$$
\tilde{v}_{T k}=\int d^{2} \boldsymbol{u} \tilde{A}\left(\boldsymbol{u}_{k}-\boldsymbol{u}\right) \tilde{T}(\boldsymbol{u}) e^{2 \pi i \boldsymbol{u} \cdot \boldsymbol{\theta}_{k}}
$$

where $\boldsymbol{\theta}_{k}$ is the pointing direction of the array when data point $k$ was taken. A frequency dependent calibration factor to convert between the units of the visibilities (flux density in Jy) and the units of the CMB temperature field has been absorbed into $\tilde{A}$. The $\boldsymbol{u}=(u, v)$ is the coordinate system in the aperture plane (also called the uv-plane). Equivalently, the $\boldsymbol{k}$-vectors of the plane-wave decomposition of the sky represented by the Fourier transform are given by $\boldsymbol{k}=2 \pi \boldsymbol{u}$. Note that these Fourier modes can be considered as the small angle $<10^{\circ}$ limit of the spherical harmonics used in full-sky decomposition of the CMB. In this case the multipole $\ell=|\boldsymbol{k}|=2 \pi|\boldsymbol{u}|$, the magnitude of the $\boldsymbol{k}$ vector. Our Fourier convention is that $\tilde{T}$ is the inverse Fourier transform of the sky temperature distribution, or

$$
\tilde{T}(\boldsymbol{u})=\int d^{2} \boldsymbol{\theta} T(\boldsymbol{\theta}) F^{-1}(\boldsymbol{u}, \boldsymbol{\theta}) \quad F^{-1}=e^{-2 \pi i \boldsymbol{u} \cdot \boldsymbol{\theta}} \Leftrightarrow F=e^{2 \pi i \boldsymbol{u} \cdot \boldsymbol{\theta}} .
$$

Since $T$ is real, the transform $\tilde{T}$ is Hermitian in the complex uv-plane and only the real and imaginary parts over a half-plane are independent.

Our interferometer sums the sky signal over all relative pointing directions $\theta$ with phase in the aperture plane given above. The wavefronts are modified by the aperture illumination functions of the individual antennas, and thus $\tilde{\mathbf{A}}$ is the aperture cross-correlation of the aperture functions of the pair of antennas involved in the correlation. Note that the center of the correlation $k$ lies at $\boldsymbol{u}_{k}=\boldsymbol{B}_{i j} / \lambda_{k}$ and has a width equal to the sum of the dish diameters in wavelengths, with no support outside this region of the uv-plane: $\tilde{A}_{i j}\left(\boldsymbol{u}^{\prime}\right)=0$ for $\left|\boldsymbol{u}^{\prime}\right| \geq\left(D_{i}+D_{j}\right) / \lambda_{k}$ for a visibility between antennas $i$ and $j$ with diameters $D_{i}$ and $D_{j}$. observed at wavelength $\lambda_{k}$. This is illustrated in Figure 2. Note that the Fourier transform $A$ of $\tilde{A}$ is traditionally known as the primary beam response on the sky, and defines the field of view of the interferometer.

We turn this into a linear algebraic matrix operation $\tilde{\boldsymbol{v}}_{T}=\tilde{\mathbf{A}}_{T} \tilde{\boldsymbol{t}}+\tilde{\boldsymbol{n}}_{T}$, where 

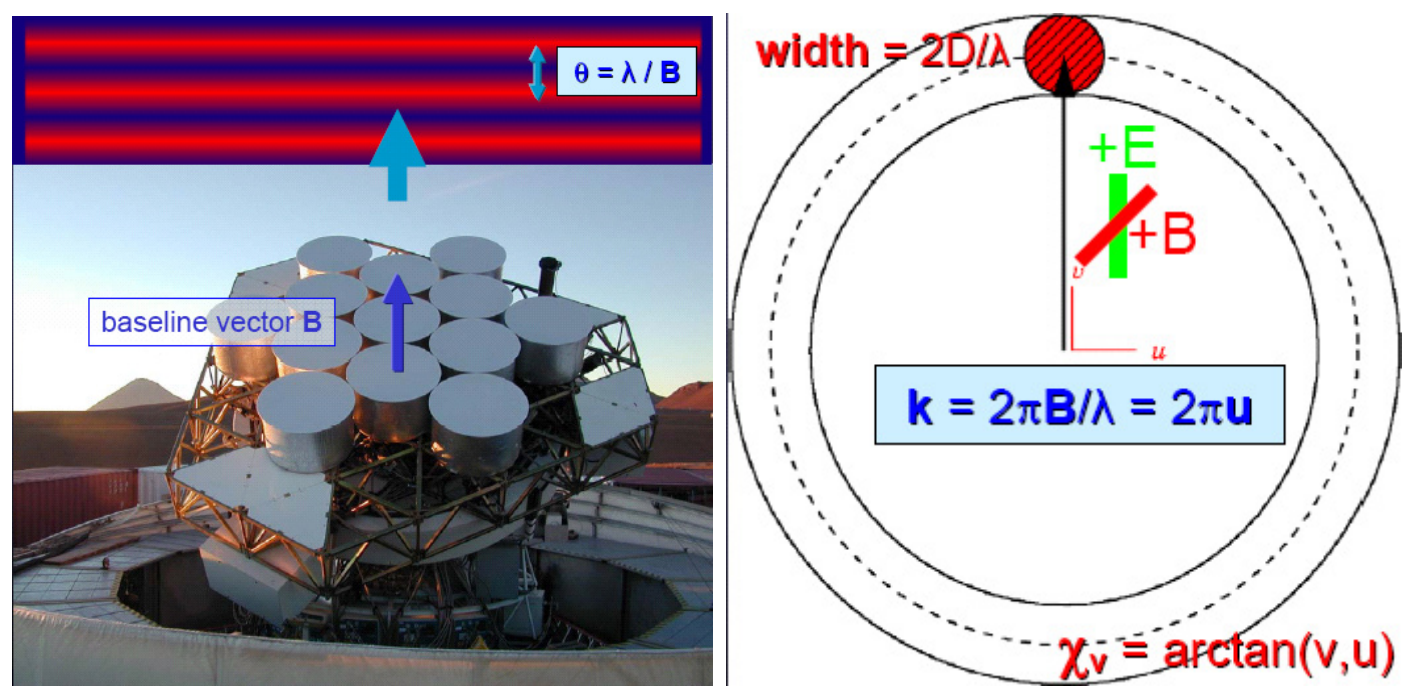

Fig. 2. Left: The physical baseline between a pair of CBI antennas to be correlated. If a correlated signal were transmitted from the pair, it would paint a sinusoidal fringe on the sky with angular wavelength $B / \lambda$. This is equivalent to the plane wave that this baseline is sensitive to. Right: In the uv-plane, the baseline is the center of a locus of points of width equal to the sum of the dish diameters in wavelengths. The correlation sums together the complex coefficients of the plane waves whose $\boldsymbol{k}$-vectors $\boldsymbol{k}=2 \pi \boldsymbol{u}$ fall in this region. For polarization, the $E$ and $B$ modes are defined with respect to the orientation of the $\boldsymbol{k}$-vectors in uv-space.

temperature signal vector $\tilde{t}_{l}=\tilde{T}\left(\boldsymbol{u}_{l}\right)$ for uv-plane "cell" $\left(u_{l}, v_{l}\right)$. The elements of $\tilde{\mathbf{A}}_{T}$ contain the interferometer phase factor of Equation 6, and in matrix form

$$
\tilde{A}_{T k l}=\tilde{A}_{T}\left(\boldsymbol{u}_{k}-\boldsymbol{u}_{l}\right) e^{2 \pi i \boldsymbol{u}_{l} \cdot \boldsymbol{\theta}_{k}}
$$

where $\tilde{A}_{T}$ is the aperture cross-correlation function appropriate for the Stokes I intensity. Thus $\tilde{\mathbf{A}}_{T}$ has dimensions of number of visibilities times number of cells $l$ in uv-space.

The operation described in in Equation 4 "grids" the visibilities into the uvplane using $\tilde{\mathbf{H}}$ as a kernel. If we use $\tilde{\mathbf{A}}_{T}$ to form $\tilde{\mathbf{H}}_{T}$, then we get the gridded temperature estimator

$$
\tilde{\boldsymbol{d}}_{T}=\tilde{\mathbf{H}}_{T} \tilde{\boldsymbol{v}}_{T}=\tilde{\mathbf{R}}_{T} \tilde{\boldsymbol{t}}+\tilde{\boldsymbol{n}}_{\boldsymbol{d}_{T}} \quad \tilde{\mathbf{R}}_{T}=\tilde{\mathbf{H}}_{T} \tilde{\mathbf{A}}_{T} \quad \tilde{\boldsymbol{n}}_{\boldsymbol{d}_{T}}=\tilde{\mathbf{H}}_{T} \tilde{\boldsymbol{n}}_{T}
$$

For purposes of CBI analysis, we carried out this gridding procedure on a regular lattice $\left(u_{l}, v_{l}\right)$ as described in (14). In our pipeline described in (14), the singularity problems with $\tilde{\mathbf{R}}_{T}$ and $\tilde{\mathbf{N}}_{\boldsymbol{d}_{T}}$ were handled by truncating the gridded data vector $\tilde{\boldsymbol{d}}$ by selecting every nth grid cell in (u.v) and to throw out zero-weight cells (this pruned version of $\tilde{\boldsymbol{d}}_{T}$ was called $\boldsymbol{\Delta}$ in that paper). 
The polarization of the radiation field can be included by considering a signal vector $\tilde{\boldsymbol{p}}$ for the polarization field $\tilde{P}(\boldsymbol{u})$ in uv-space

$$
\tilde{P}(\boldsymbol{u})=\tilde{Q}(\boldsymbol{u})+i \tilde{U}(\boldsymbol{u})=[\tilde{E}(\boldsymbol{u})+i \tilde{B}(\boldsymbol{u})] e^{i 2 \chi(\boldsymbol{u})}
$$

where $\chi(\boldsymbol{u})=\tan ^{-1}(v / u)$ is the angle of the plane wave $\boldsymbol{k}$-vector $\boldsymbol{k}=2 \pi \boldsymbol{u}$ on the sky. The $Q$ and $U$ fields are the usual linearly polarized Stokes parameters representing components of a polarization (pseudo-)vector in the frame of the sky, with $Q$ aligned North $(Q>0)$ or East $(Q<0)$ and $U$ at $\pm \pi / 4$ with respect to North (NE/NW). The $E$ and $B$ mode decomposition, e.g. (19), is in the frame of the plane wave decomposition of the sky in the Fourier domain. Thus, an $E$-mode plane wave has a linear polarization vector aligned along $(E>0)$ or perpendicular to $(E<0)$ the wave $\boldsymbol{k}$-vector, and a $B$-mode has its polarization at $\pm \pi / 4$ to the wave. See the right panel of Figure 2 for a graphical illustration of this. Note that the $\tilde{P}$ field is defined over the entire complex uv-plane and is not Hermitian. As in the definition of $\tilde{\boldsymbol{t}}$, we can define a polarization vector $\tilde{\boldsymbol{p}}$

$$
\tilde{\boldsymbol{p}}=\tilde{\mathbf{X}}[\tilde{\boldsymbol{e}}+i \tilde{\boldsymbol{b}}] \quad \tilde{X}_{l l^{\prime}}=e^{i 2 \chi\left(\boldsymbol{u}_{l}\right)} \delta_{l l^{\prime}} \quad \tilde{e}_{l}=\tilde{E}\left(\boldsymbol{u}_{l}\right) \quad \tilde{b}_{l}=\tilde{B}\left(\boldsymbol{u}_{l}\right)
$$

where $\tilde{p}_{l}=\tilde{P}\left(\boldsymbol{u}_{l}\right)$.

The cross-polar (RL,LR) correlation products are sensitive to $\tilde{P}$. We let $\tilde{\boldsymbol{v}}_{P}=$ $\tilde{\mathbf{A}}_{P} \tilde{\boldsymbol{p}}+\tilde{\boldsymbol{n}}_{P}$, with

$$
\tilde{A}_{P k l}=\tilde{A}_{P}\left(\boldsymbol{u}_{k}-\boldsymbol{u}_{l}\right) e^{2 \pi i \boldsymbol{u}_{l} \cdot \boldsymbol{\theta}_{k}} e^{-i 2\left(\chi\left(\boldsymbol{u}_{k}\right)-\chi_{i j 0}\right)}
$$

where $\chi\left(\boldsymbol{u}_{k}\right)$ is the baseline orientation of visibility $k$ and $\chi_{i j 0}$ is the reference orientation of baseline $i j$ in the array (e.g. at deck-angle zero for a planar array, or at transit for a tracking array). This last phase factor is there to correct for the parallactic angle orientation of the array feeds on the sky, which are locked to the deck of our planar array and thus rotate with the $\left(u_{k}, v_{k}\right)$ of the visibilities of a given baseline. The visibilities are calibrated such that $\delta \chi=\chi\left(\boldsymbol{u}_{k}\right)-\chi_{i j 0}=0$ at deck angle zero. The $\tilde{\boldsymbol{v}}_{P}$ is thus equivalent to an RL correlation product, and LR visibilities should be treated as RL correlations complex-conjugated and reflected about the origin in the uv-plane. Note that most radio interferometers apply the parallactic angle rotation (the last term on the right hand side of Equation 12) on-line so that the visibility data can be transformed easily to make $Q$ and $U$ Stokes images in the standard software packages. For CBI this is done also in our calibrated visibility data, but before gridding we do the de-rotation so that the visibilities are in the frame of $\tilde{E}$ and $\tilde{B}$ (see below). Also, the polarization beam transform $\tilde{A}_{P}$ is in general different than $\tilde{A}$ for the intensity, though for a symmetric optical system like 
that used on CBI, and neglecting leakage (see below), they should be almost identical.

In the more familiar integral form,

$$
\begin{aligned}
\tilde{v}_{P k}= & \int \mathrm{d}^{2} \boldsymbol{u}[\tilde{E}(\boldsymbol{u})+i \tilde{B}(\boldsymbol{u})] e^{2 \pi i \boldsymbol{u} \cdot \boldsymbol{\theta}_{k}} e^{i 2 \chi_{i j 0}} \\
& \times \tilde{A}_{P}\left(\boldsymbol{u}_{k}-\boldsymbol{u}\right) e^{i 2\left(\chi(\boldsymbol{u})-\chi\left(\boldsymbol{u}_{k}\right)\right)}+\tilde{n}_{P k}
\end{aligned}
$$

The last terms in the integral represent the smearing in uv-space due to the macroscopic size of the apertures, which mix the $E$ and $B$ modes. If our interferometer array elements were infinitely small and thus sensitive to the entire sky, then $\tilde{\mathbf{A}}_{P}$ would be a delta-function and our interferometer would recover $\tilde{E}+i \tilde{B}$ directly. As it is, it is straightforward to recover a filtered version of $\tilde{E}+i \tilde{B}$ and thus subsequent CMB polarization analysis is relatively easy using gridded polarization data.

As in the case for $\tilde{\boldsymbol{d}}_{T}$, we can take the $\tilde{\mathbf{A}}_{P}$ and $\tilde{\boldsymbol{v}}_{P}$ and for the gridded polarization estimators

$$
\tilde{\boldsymbol{d}}_{P}=\tilde{\mathbf{H}}_{P} \tilde{\boldsymbol{v}}_{P}=\tilde{\mathbf{R}}_{P} \tilde{\boldsymbol{p}}+\tilde{\boldsymbol{n}}_{\boldsymbol{d}_{P}} \quad \tilde{\mathbf{R}}_{P}=\tilde{\mathbf{H}}_{P} \tilde{\mathbf{A}}_{P} \quad \tilde{\boldsymbol{n}}_{\boldsymbol{d}_{P}}=\tilde{\mathbf{H}}_{P} \tilde{\boldsymbol{n}}_{P}
$$

However, for the CMB, it is more convenient if we work in terms of $E$ and $B$, and thus we write

$$
\tilde{\boldsymbol{d}}_{P}=\tilde{\mathbf{R}}_{E} \tilde{\boldsymbol{e}}+\tilde{\mathbf{R}}_{B} \tilde{\boldsymbol{b}}+\tilde{\mathbf{H}}_{P} \tilde{\boldsymbol{n}}_{P} \quad \tilde{\mathbf{R}}_{E}=\tilde{\mathbf{R}}_{P} \tilde{\mathbf{X}} \quad \tilde{\mathbf{R}}_{B}=i \tilde{\mathbf{R}}_{P} \tilde{\mathbf{X}}
$$

where the elements of $\tilde{\mathbf{X}}$ are defined in Equation 11 .

Full Polarization Processing: For processing, we assemble a single long gridded data vector and a concatenated visibility vector $\tilde{\boldsymbol{d}}=\tilde{\mathbf{H}} \tilde{\boldsymbol{v}}$, with

$$
\left(\begin{array}{c}
\tilde{\boldsymbol{d}}_{T} \\
\tilde{\boldsymbol{d}}_{P}
\end{array}\right)=\left(\begin{array}{cc}
\tilde{\mathbf{H}}_{T} & 0 \\
0 & \tilde{\mathbf{H}}_{P}
\end{array}\right)\left(\begin{array}{c}
\tilde{\boldsymbol{v}}_{T} \\
\tilde{\boldsymbol{v}}_{P}
\end{array}\right) .
$$

Then, combining Equations 9 and 15 we get

$$
\tilde{\boldsymbol{d}}=\tilde{\mathbf{R}}_{t} \tilde{\boldsymbol{t}}+\tilde{\mathbf{R}}_{e} \tilde{\boldsymbol{e}}+\tilde{\mathbf{R}}_{b} \tilde{\boldsymbol{b}}+\tilde{\boldsymbol{n}}_{d}
$$

where

$$
\tilde{\mathbf{R}}_{t}=\left(\begin{array}{c}
\tilde{\mathbf{R}}_{T} \\
0
\end{array}\right) \quad \tilde{\mathbf{R}}_{e}=\left(\begin{array}{c}
0 \\
\tilde{\mathbf{R}}_{E}
\end{array}\right) \quad \tilde{\mathbf{R}}_{b}=\left(\begin{array}{c}
0 \\
\tilde{\mathbf{R}}_{B}
\end{array}\right) .
$$


The noise vector is given by

$$
\tilde{\boldsymbol{n}}_{d}=\left(\begin{array}{cc}
\tilde{\mathbf{H}}_{T} & 0 \\
0 & \tilde{\mathbf{H}}_{P}
\end{array}\right)\left(\begin{array}{c}
\tilde{\boldsymbol{n}}_{T} \\
\tilde{\boldsymbol{n}}_{P}
\end{array}\right)=\left(\begin{array}{c}
\tilde{\mathbf{H}}_{T} \\
\tilde{\boldsymbol{n}}_{T} \\
\tilde{\mathbf{H}}_{P} \tilde{\boldsymbol{n}}_{P}
\end{array}\right) .
$$

Covariances: The visibility covariances contain the information about the CMB power spectrum, and that of any foregrounds and the instrumental noise. The covariance between co-polar visibilities measures the $T T$ power spectrum $C_{\ell}$. Covariances between cross-polar visibilities measure $E E$ and $B B$ (as well as $E B)$. Covariances between co-polar and cross-polar visibilities measure the $T E$ (and $T B$ ) power spectrum. Thus,

$$
\mathbf{C}=\left\langle\tilde{\boldsymbol{d}} \tilde{\boldsymbol{d}}^{\mathrm{t}}\right\rangle=\tilde{\mathbf{N}}+\mathbf{C}^{T T}+\mathbf{C}^{E E}+\mathbf{C}^{B B}+\mathbf{C}^{T E}+\ldots
$$

where we must include all known noise and signal correlations, including foregrounds such a point sources and ground signal (see below).

Because the fields $T, E, B$ are real and thus have Hermitian transforms, we consider the two covariance components between the complex signal vectors:

$$
\mathbf{C}^{s s^{\prime}}=\left\langle\tilde{s} \tilde{\boldsymbol{s}}^{\prime \dagger}\right\rangle \quad \overline{\mathbf{C}}^{s s^{\prime}}=\left\langle\tilde{\boldsymbol{s}} \tilde{\boldsymbol{s}}^{\prime \mathrm{t}}\right\rangle
$$

with $s, s^{\prime}=t, e, b$, and $\dagger$ denotes complex conjugation and transpose for a complex vector. These are related to the corresponding power and cross-power spectra $C_{\ell}^{S S^{\prime}}$ through

$$
C_{l l^{\prime}}^{s s^{\prime}}=C_{\ell}^{S S^{\prime}} \delta^{2}\left(\boldsymbol{u}_{l}-\boldsymbol{u}_{l^{\prime}}\right) \quad \bar{C}_{l l^{\prime}}^{s s^{\prime}}=C_{\ell}^{S S^{\prime}} \delta^{2}\left(\boldsymbol{u}_{l}+\boldsymbol{u}_{l^{\prime}}\right)
$$

for $\ell=2 \pi\left|\boldsymbol{u}_{l}\right|$ and $S, S^{\prime}=T, E, B$. Note that covariance block between real and imaginary parts of complex quantities $z$ and $z^{\prime}$ is given by

$$
\left(\begin{array}{cc}
\left\langle\operatorname{Re} z \operatorname{Re} z^{\prime}\right\rangle & \left\langle\operatorname{Re} z \operatorname{Im} z^{\prime}\right\rangle \\
\left\langle\operatorname{Im} z \operatorname{Re} z^{\prime}\right\rangle & \left\langle\operatorname{Im} z \operatorname{Im} z^{\prime}\right\rangle
\end{array}\right)=\left(\begin{array}{cc}
\frac{1}{2} \operatorname{Re}[C+\bar{C}] & -\frac{1}{2} \operatorname{Im}[C-\bar{C}] \\
\frac{1}{2} \operatorname{Im}[C+\bar{C}] & \frac{1}{2} \operatorname{Re}[C-\bar{C}]
\end{array}\right)
$$

where $C=\left\langle z z^{\prime *}\right\rangle$ and $\bar{C}=\left\langle z z^{\prime}\right\rangle$. Thus, the blocks of $\mathbf{C}^{S S^{\prime}} S, S^{\prime}=T, E, B$ can beconstructed using $\tilde{\mathbf{R}}_{s} \mathbf{C}^{s s^{\prime}} \tilde{\mathbf{R}}_{s^{\prime}}^{\dagger}$ and $\tilde{\mathbf{R}}_{s} \overline{\mathbf{C}}^{s s^{\prime}} \tilde{\mathbf{R}}_{s^{\prime}}^{\mathrm{t}}$, while the noise covariance matrix $\tilde{\mathbf{N}}$ is built from $\left\langle\boldsymbol{n} \boldsymbol{n}^{\dagger}\right\rangle$ and $\left\langle\boldsymbol{n} \boldsymbol{n}^{\mathrm{t}}\right\rangle$ - see $\S 6.1$ of (14) for details.

Imaging: One can construct dirty images $\boldsymbol{d}_{T}$ and $\boldsymbol{d}_{P}$ using Equation 5, with the elements of $\mathbf{F}$ given in Equation 7 . Note that the dirty images have an 
extra primary beam attenuation factor as compared to a standard radio interferometer dirty image (which leaves out this factor), due to the presence of $\tilde{\mathbf{A}}^{\mathrm{t}}$ or approximate convolutional kernel in $\tilde{\mathbf{H}}_{T}$. This factor is necessary in order to optimally weight a mosaiced image where different visibilities come from a raster of different pointings $\boldsymbol{\theta}_{k}$. This does mean that signals at the outside of the image are attenuated by the square of the primary beam instead of just by the primary beam. If desired, this can be corrected for as this is a known function.

One can construct a vector for the response to a point source at a fiducial phase center $\boldsymbol{\theta}_{0}$

$$
\tilde{d}_{T 0 i}=\tilde{H}_{T i k} e^{2 \pi i \boldsymbol{u} \cdot\left(\boldsymbol{\theta}_{k}-\boldsymbol{\theta}_{0}\right)} .
$$

The map $\boldsymbol{d}_{T 0}$ corresponding to the vector $\tilde{\boldsymbol{d}}_{T 0}$ is equivalent to the pointspread function described earlier. These normalizations can be used to give a calibrated images.

One can also construct a Wiener filtered version of the dirty image of Equation 5, for example

$$
\boldsymbol{d}_{X}=\mathbf{F} \tilde{\boldsymbol{d}}_{X}=\mathbf{M}_{X} \boldsymbol{d}_{T} \quad \tilde{\boldsymbol{d}}_{X}=\tilde{\mathbf{M}}_{X} \tilde{\boldsymbol{d}}_{T}
$$

is a filtered temperature map using Fourier filter $\tilde{\mathbf{M}}_{X}$ whose corresponding image plane filter is $\mathbf{M}_{X}=\mathbf{F} \tilde{\mathbf{M}}_{X} \mathbf{F}^{-1}$. The $\tilde{\boldsymbol{d}}_{X}$ can be normalized by the filtered normalization $\tilde{\mathbf{M}}_{X} \tilde{\boldsymbol{d}}_{T 0}$. In $\S 8$ of (14) we described imaging using a Wiener filter $\tilde{\mathbf{M}}_{X}=\mathbf{C}_{X} \mathbf{C}^{-1}$ built from the covariance matrices and demonstrated their use on the gridded temperature estimators $\tilde{\boldsymbol{d}}_{T}$.

\section{Application to the Cosmic Background Imager}

The CBI: The Cosmic Background Imager (CBI) is described in the presentation by Tim Pearson (this conference), and in (9). The CBI is an interferometer array located at the high altitude $(5000 \mathrm{~m})$ ALMA site in the Northern Chilean Andes near San Pedro de Atacama. The CBI operated from Jan 2000 to May 2005, and during this period the salient characteristics of the CBI were:

- operating frequencies $26-36 \mathrm{GHz}$ (wavelengths $1.15-0.83 \mathrm{~cm}$ )

- 13 antennas, $90 \mathrm{~cm}$ diameter $(78-108 \lambda)$

- antennas mounted on a $6 \mathrm{~m}$ planar platform (reconfigurable)

- 78 baselines (between 13 antennas) from $1 \mathrm{~m}$ to $5.5 \mathrm{~m}$

- 10 frequency channels, $1 \mathrm{GHz}$ wide 
- 780 complex correlations (baselines $\times$ channels)

- primary field-of-view $45^{\prime}$ at $30 \mathrm{GHz}$

- single polarization ( $\mathrm{R}$ or $\mathrm{L}$ ) each element, switchable

- low polarization leakage $(<2 \%)$

From 2000-2002, the CBI was used to observe the temperature anisotropies in the CMB, as reported in (20), (21), (22), (23). All but one of the 13 antennas were set to receive the same polarization, with the 12 baselines to the crosspolarized element used to test out polarization observations for the Caltech $\mathrm{PhD}$ thesis of John Cartwright, reported in (24). For these observations, we placed the antennas in a spread configuration which contained baseline lengths from $1 \mathrm{~m}$ to the maximum $5.5 \mathrm{~m}$ (reaching $\ell_{\max }=2 \pi B_{\max } / \lambda_{\min } \approx 4200$ ). From 2002-2005 we observed with split polarization ( $7 \mathrm{~L}$ and $6 \mathrm{R}$ ) and with the antennas in the closest packed configuration to maximize sensitivity, with the results reported in (25) and (26).

We now describe some of the imaging issues involved with analysis of the CBI data.

Ground signal: Because of the short baselines $(\sim 100 \lambda)$, the CBI is sensitive to radiation coming from the ground, in particular the higher mountain peaks on the horizon around the CBI site. This signal is constant on short timescales for a given orientation of the array with respect to the ground (e.g. in azimuthelevation coordinates). For the temperature observations from 2000-2002, we observed pairs of fields at the same declination separated by $8^{\mathrm{m}}$ in right ascension (or $2^{\circ}$ apart on the sky) for 8 minutes each, thus tracking through the same azimuth and elevation. The visibilities from these pairs were then differenced before imaging and analysis, removing the common-mode ground signal. This meant that we could not distinguish a positive signal in the main field from a negative signal in the trail field, for example to determine whether signals were due to the Sunyaev-Zeldovich effect (27). The differencing also reduced our efficiency by $50 \%$ (by keeping the difference but throwing away the sum). We took care of the effect of the differencing on power-spectrum estimation by including it in the calculation of $\tilde{\mathbf{A}}$ used in $\tilde{\mathbf{R}}$ as described in $\S 6.6$ of (14).

For the polarization observations of 2002-2005, we observed a constant declination strip of 6 fields separated by $3^{\mathrm{m}}$ in right ascension (45' apart on sky) and projected out a constant scan mean during the likelihood calculation. This increased efficiency by effectively only dropping a single mean out of the six fields and allows for better localization of signals.

Point sources: The most serious astronomical foreground signal that affects our CMB measurements is that due to unresolved "point" sources, mostly radio galaxies and quasars. Unfortunately, we did not have the simultaneous 
$30 \mathrm{GHz}$ measurements of the flux densities of these sources needed to accurately subtract the effects from the data. However, we do know the locations of most of the sources that could plausibly contaminate the CBI observations from the 1.4 GHz NRAO-VLA Sky Survey (NVSS), (28). The positions were used to construct a source covariance matrix $\mathbf{C}_{s r c}$ which in turn was used to project out the contributions of these sources during the power spectrum analysis and imaging. The remaining faint radio sources were treated as a Gaussian random foreground noise field and accounted for using a covariance matrix $\mathbf{C}_{\text {res }}$. This procedure was also described in $\S 6.3-6.4$ of (14).

Polarization Leakage: In practice, radio receivers have imperfect polarization response. For example, a detector nominally sensitive only to right circular polarization $(R)$ will also pick up a small amount of left circular polarization $(L)$. This means that the correlation products are also impure, and the projectors $\tilde{\mathbf{R}}_{t}, \tilde{\mathbf{R}}_{e}$, and $\tilde{\mathbf{R}}_{b}$, in Equation 18 pickup non-zero cross-terms between $\tilde{\boldsymbol{d}}_{T}$ and $\tilde{\boldsymbol{d}}_{P}$, thus we can generalize to

$$
\tilde{\mathbf{R}}_{t}=\left(\begin{array}{c}
\tilde{\mathbf{R}}_{T} \\
\tilde{\mathbf{R}}_{P t}
\end{array}\right) \quad \tilde{\mathbf{R}}_{e}=\left(\begin{array}{c}
\tilde{\mathbf{R}}_{T e} \\
\tilde{\mathbf{R}}_{E}
\end{array}\right) \quad \tilde{\mathbf{R}}_{b}=\left(\begin{array}{c}
\tilde{\mathbf{R}}_{T b} \\
\tilde{\mathbf{R}}_{B}
\end{array}\right)
$$

where the small leakage projectors $\tilde{\mathbf{R}}_{P t}, \tilde{\mathbf{R}}_{T e}$ and $\tilde{\mathbf{R}}_{T b}$ encapsulate these errors. After this, the analysis can proceed as normal.

Foregrounds: In the absence of foregrounds, the response of the visibilities to the CMB, for example in Equation 1, is pure. If there is a foreground field with transform $\tilde{\boldsymbol{f}}$, then

$$
\tilde{\boldsymbol{v}_{T}}=\tilde{\mathbf{A}}_{T} \tilde{\boldsymbol{t}}+\mathbf{G}_{f}^{-1} \tilde{\mathbf{A}}_{T} \tilde{\boldsymbol{f}}+\tilde{\boldsymbol{n}}_{T}
$$

where $\mathbf{G}_{f}$ is a diagonal matrix containing the difference in frequency spectrum (per visibility) between the foreground and the CMB. The conversion between the units of the visibilities and the CMB field given by the thermal blackbody spectrum is contained in $\tilde{\mathbf{A}}_{T}$. Bandpowers for $\left\langle\tilde{\boldsymbol{f}} \tilde{\boldsymbol{f}}^{\dagger}\right\rangle$ can be solved for in addition to the CMB power spectrum.

One can also apply a non-thermal frequency filter $\tilde{\mathbf{H}}_{f}=\tilde{\mathbf{H}} \mathbf{G}_{f}$ during gridding which will optimize response to that foreground in order to image or assess the contamination level in the data. In the presence of unknown foregrounds, one can apply a general first-order filter sensitive to a deviation from a thermal spectrum, such as a logarithmic slope $G_{f}\left(\nu_{k}\right)=\ln \left(\nu_{k} / \nu_{0}\right)$ where $\nu_{0}$ is a reference frequency in the center of the band $(31 \mathrm{GHz}$ for $\mathrm{CBI})$. This technique is known in radio interferometry as multi-frequency synthesis and is used to map out the frequency spectrum as well as intensity of the sky (29). 
Maximum Likelihood, Power Spectrum Estimation, and Projection: Given the covariance matrix, we can compute the log-likelihood

$$
\ln \mathcal{L}=\frac{1}{2} \ln \left(\operatorname{det} \mathbf{C}^{-1} / 2 \pi\right)-\frac{1}{2} \tilde{\boldsymbol{d}}^{\mathrm{t}} \mathbf{C}^{-1} \tilde{\boldsymbol{d}}
$$

If we parameterize the terms in Equation 20 by amplitudes and shapes, or break into "bands" in $\ell$ with "bandpowers" $q_{B}^{s s '}(30)$, e.g. $\mathbf{C}^{T T}=\sum_{B} q_{B}^{T T} \mathbf{C}_{B}^{T T}$. These bandpowers are the amplitudes of the power spectrum in a given bin in $\ell$ relative to the assumed shape $\mathbf{C}_{B}^{s s}$, generally taken to be "flat" $\mathbf{C}_{\ell}^{T T}=2 \pi / \ell^{2}$. One can then solve for the bandpowers that maximize $\ln \mathcal{L}\left(\boldsymbol{q}^{T T}, \boldsymbol{q}^{E E}, \boldsymbol{q}^{B B}, \boldsymbol{q}^{T E}\right)$.

Likewise, one can downweight nuisance modes in the data due to contamination by point source foregrounds or ground emission if the shape of the covariance matrices are known. If terms such as $q^{\text {ground }} \mathbf{C}^{\text {ground }}$ and $q^{\text {src }} \mathbf{C}^{\text {src }}$ are added to $\mathbf{C}$, and the pre-factors $q^{\text {ground }}$ and $q^{s r c}$ set to arbitrarily high values, then the corresponding modes in $\mathbf{C}^{-1}$ are downweighted and do not contribute to the likelihood. This technique is described in $\S 6$ of (14).

The CBI Pipeline: The analysis of CBI polarimetry data is carried out using a pipeline running on the 512-processor McKenzie Cluster ${ }^{2}$ at the Canadian Institute for Theoretical Astrophysics (CITA). The main part of the pipeline software was implemented in two parallel codes, mpigridr, which does the gridding and covariance matrix calculation given an input set of (millions of) visibilities, and mpilikely, which takes the (thousand or so) gridded estimators and carries out the maximum likelihood power spectrum estimation procedure and related tasks. It took on order of 6 hours on McKenzie to process all of the polarization data and produce the power spectra for (26) (excluding the calculation of window functions).

E/B Imaging: The construction of images from the gridded CBI data was carried out separately, using the output of mpigridr. This involves making a filtered version of the dirty image given in Equation 25. In (26) we presented $T, E$, and $B$ maps in both the uv-plane and the image plane made using this method, employing a filter $\tilde{\mathbf{M}}_{X}=\mathcal{B} \tilde{\mathbf{R}}_{\text {eff }}^{-1} \mathbf{C}_{T} \mathbf{C}^{-1}$ where $\tilde{\mathbf{R}}_{\text {eff }}^{-1}$ is an approximation to the singular "true" $\tilde{\mathbf{R}}^{-1}$ and $\mathcal{B}$ a smoothing kernel. SeeFigure 3 for images of the $20 \mathrm{~h}$ field.

Comparison with VSA: In 2002-2004, the VSA observed some of the same fields observed by the CBI. As reported in (32), a variant of the gridded estimator analysis was used to demonstrate agreement between the two datasets. The CBI visibilities were gridded with the VSA aperture function and the VSA visibilities gridded with the CBI aperture function: e.g. $\tilde{\mathbf{H}}_{C B I}=\tilde{\mathbf{A}}_{V S A}^{\dagger} \tilde{\mathbf{N}}_{C B I}^{-1}$

$\overline{2}$ CITA's McKenzie cluster, (31), was funded by the Canada Foundation for Innovation and the Ontario Innovation Trust. 

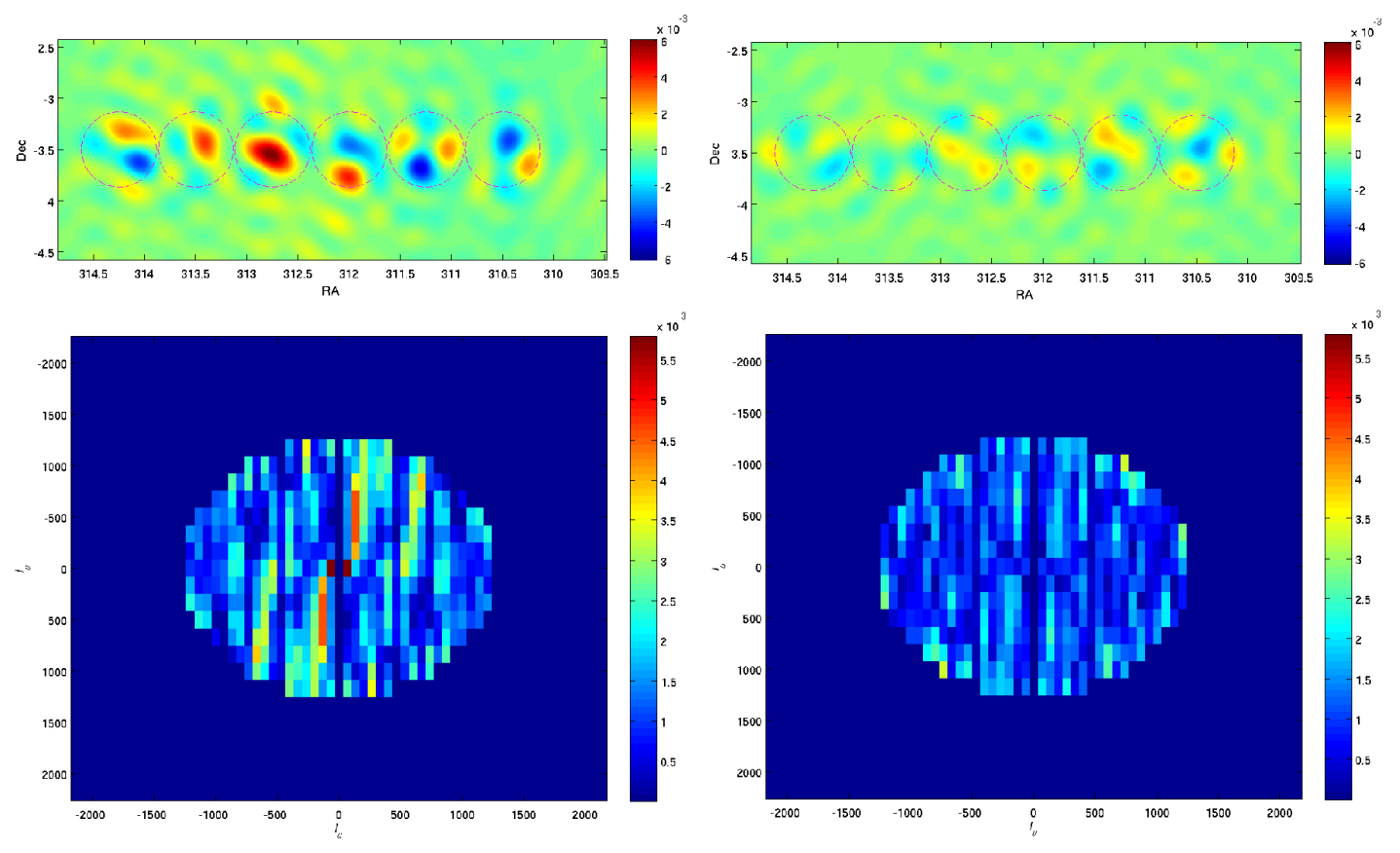

Fig. 3. Top row ( $L$ to $R$ ): Images $\boldsymbol{d}$ of the $E$ and $B$ fields on the sky using the CBI $20 \mathrm{~h}$ strip data as presented in (26). The color bar shows the flux density scale in $\mathrm{mJy} /$ beam. The six dashed circles are the half-power points of the mosaic fields in that strip, with offsets given in degrees on the coordinate axes. A common-mode signal along the scan direction has been projected out. Bottom row ( $L$ to $R$ ): The uv-plane maps $\tilde{\boldsymbol{d}}$ for $E$ and $B$ respectively, corresponding to the sky images in the top row. The maps show the amplitudes of estimators in cells in uv-space, and have been truncated at $\ell=1200$. Coordinate axes are $k_{u}, k_{v}$ in units of $\ell$. Because the $20 \mathrm{~h}$ strips are oriented E-W, the resolution along the $u$ axis is higher than that along the $v$ axis. About $3 \times$ more power is seen in the $E$ uv-maps and images than in the $B$ maps and images, which in turn are consistent with noise. Uv-maps such as this can be used to search for non-Gaussianity.

and $\tilde{\mathbf{H}}_{V S A}=\tilde{\mathbf{A}}_{C B I}^{\dagger} \tilde{\mathbf{N}}_{V S A}^{-1}$. This allowed comparison of the uv-space signals and power spectra on the same footing.

\section{Future CMB Interferometry}

There are a number of CMB interferometers that have operated, are currently operating, or will be brought online in the near future. These include the special-purpose instruments AMI, AMIBA, CAT, CBI, DASI, MINT, VSA, as well as the use of the BIMA, OVRO-MMA, and Ryle telescope for CMB studies. In the future, the EVLA and ALMA will also have some capabilities of use for CMB research. In particular, with 50 12-m diameter antennas capable of being placed in a close-packed configuration, the Atacama Large Millimeter-submillimeter Array (ALMA) can provide high-sensitivity CMB 
polarimetric observations at high- $\ell$, particularly if a $30 \mathrm{GHz}$ band were installed on the array (the lowest frequency covered in currently funded receiver bands is $86 \mathrm{GHz}$ ).

Mega-pixel CMB interferometer: Given the success of CMB interferometers such as DASI and CBI at measuring the polarization power spectrum, it is natural to ask whether it is possible to scale up these instruments to build a next-generation CMB interferometer with $10^{2}-10^{3}$ elements yielding $10^{4}-10^{6}$ instantaneous baselines — a "mega-pixel" interferometric digital camera. Such an instrument would retain the interferometric advantages outlined above, such as resilience against many types of systematics and ease of analysis. Channelization of the bandwidth is also necessary in order to deal with foregrounds, a minimum of 10 channels. The correlator needed to handle $10^{5}$ $10^{7}$ baseline-channels in each of the 4 polarization correlation products is substantial. With the advances in both analog and digital technology in the decade since CBI and DASI were designed, and the development of novel large correlators underway for next generation radio arrays such as the SKA, it is foreseeable that a CMB Mega-interferometer could be built.

Space mission: Although the advantages of interferometry are most closely matched to a ground-based array, with some imagination one can contemplate a cosmic interferometer space mission. In his closing presentation, Bruce Partridge exhorts the community to unify behind a CMBpol space mission and the phased ground and balloon-based program leading to it as advocated by the Task Force. To this end, the engineers at Davros Laboratory have developed a CMB space mission concept that the community can rally behind: the DArk Lambda Explorer for Kosmogenesis (DALEK). Sadly, the prototypes ${ }^{3}$ seem to have gone missing from the lab after disintegrating several technicians...

\section{References}

[1] A.R. Thompson, J.M. Moran, \& G.W. Swenson Jr. 1986, Interferometry and Synthesis in Radio Astronomy (New York:Wiley).

[2] H.M. Martin, R.B. Partridge, \& R.T. Rood, ApJL, 240, L79 (1980).

[3] J.E. Knoke, et al., ApJ, 284, 479 (1984).

[4] E.B. Fomalont, K.I. Kellermann, \& J.V. Wall, ApJL, 277, L23 (1984).

[5] Subrahmanyan, R., Ekers, R.D., Sinclair, M., \& Silk, J., MNRAS, 263, 416 (1993).

[6] Radford, S.J.E., ApJ, 404, L33 (1993).

[7] Timbie, P.T., \& Wilkinson, D.T., ApJ, 353, 140 (1990).

[8] C. O'Sullivan, et al., MNRAS, 274, 861 (1995).

[9] S. Padin, et al., PASP, 114, 83 (2002)

$\overline{3 \text { Early }}$ prototypes can be seen at http://en.wikipedia.org/wiki/Dalek. 
[10] E.M. Leitch, et al., ApJ, 568, 28 (2002).

[11] R.A. Watson, et al., MNRAS, 341, 1057 (2003).

[12] Holzapfel, W.L., et al., ApJ, 539, 57 (2000).

[13] J.W. Fowler, et al., ApJS, 156, 1 (2005).

[14] S.T. Myers et al., ApJ, 591, 575 (2003).

[15] M.P. Hobson, \& K. Maisinger, MNRAS, 334, 569 (2002).

[16] J.P. Hamaker, J.D. Bregman, \& R.J. Sault, A\&AS, 117, 137 (1996)

[17] R.J. Sault, J.P. Hamaker, \& J.D. Bregman, A\&AS, 117, 149 (1996)

[18] J.P. Hamaker, A\&AS, 143, 515 (2000).

[19] M. Zaldarriaga, Phys. Rev. D, 64, 103001 (2001).

[20] S. Padin, APJL, 549, L1 (2001)

[21] B.S. Mason et al., ApJ, 591, 540 (2003).

[22] T.J. Pearson et al., ApJ, 591, 556 (2003).

[23] A.C.S. Readhead et al., ApJ, 609, 498 (2004)

[24] J.K. Cartwright, et al., ApJ, 623, 11 (2005).

[25] A.C.S. Readhead et al., Science, 306, 836 (2004).

[26] J.L. Sievers et al., ApJ, in press (2006) [arXiv:astro-ph/0509203].

[27] J.R. Bond et al., ApJ, 626, 12 (2005).

[28] J.J. Condon, et al., AJ, 115, 1693 (1998).

[29] J.E. Conway, T.J. Cornwell,\& P.N. Wilkinson, MNRAS, 246, 490 (1990).

[30] J.R. Bond, A.H. Jaffe, \& L. Knox, Phys. Rev. D, 57, 2117 (1998).

[31] J. Dubinski, R.J. Humble, C. Loken, U.-L. Pen, P.G. Martin, in Proc. of the 17th Annual International Symposium on High Performance Computing Systems and Applications, May 11-14, 2003 Sherbrooke, PQ (2003).

[32] N. Rajguru, et al., MNRAS, 363, 1125 (2005). 